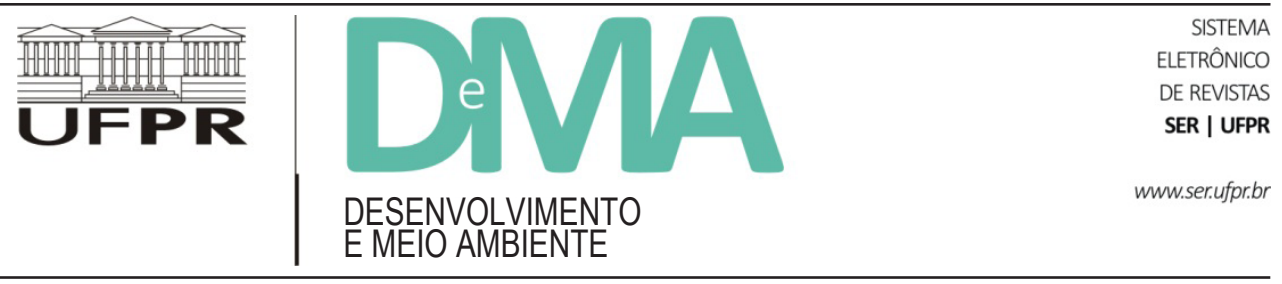

\title{
Le changement climatique entre utopie et dystopie: une analyse de la représentation du phénomène dans les presses française et brésilienne
}

\author{
Mudanças climáticas entre utopia e distopia: uma análise da \\ representação do fenômeno nas imprensas brasileira e francesa
}

\section{Climate Change Between Utopia and Dystopia: An Analysis of the Representation of the Phenomenon in French and Brazilian Newspapers}

\author{
Ana Carolina Lins PELIZ ${ }^{*}$ \\ ${ }^{1}$ Paris Sorbonne Paris IV - Celsa, France. \\ *Contact: anapeliz@gmail.com
}

Article reçu le 30 octobre 2016, version finale acceptée le 16 février 2017.

RÉSUMÉ : L'article présente les résultats d'une analyse discursive des journaux Le Monde, Le Figaro, O Estado de São Paulo et $O$ Globo au sujet de la représentation du changement climatique. L'hypothèse heuristique fil conducteur de l'étude est que la représentation du phénomène dans les journaux analysés se partage entre idées utopiques et dystopiques. Le cadre théorique trouve ses sources dans des travaux philosophiques ainsi que dans des études littéraires sur l'utopie et la dystopie. L'analyse du discours est basée sur les travaux de Sophie Moirand sur la presse quotidienne nationale française, ainsi que sur le concept de "moment discursif". Nous observons que la représentation du changement climatique dans les journaux analysés se structure autour de l'idée d'un consensus tant scientifique que politique, de la promotion des initiatives individuelles, ainsi que de la préservation de la nature et de l'usage des technologies comme réponse au problème climatique.

Mots-clés: changement climatique; presse; représentation; utopie; dystopie; analyse du discours.

RESUMO: $\quad$ O artigo apresenta os resultados de uma análise do discurso dos jornais Le Monde, Le Figaro, O Estado de São Paulo e $O$ Globo sobre a representação das mudanças climáticas. A hipótese heurística que conduz a pesquisa é de que a representação do fenômeno nos jornais se divide entre ideias utópicas e distópicas. O quadro teórico baseia-se em trabalhos filosóficos e em estudos literários sobre utopia e distopia. A análise do discurso é fundamentada nos trabalhos sobre a imprensa diária francesa realizados por Sophie Moirand e no conceito de "momento discursivo". Observa-se que a representação das mudanças climáticas nos jornais analisados estrutura-se sobre a ideia de consenso científico e político, promovendo iniciativas individuais, o ideal de preservação da natureza e o uso de tecnologias como resposta ao problema climático.

Palavras-chave: mudanças climáticas; imprensa; representação; utopia; distopia; análise do discurso. 
ABSTRACT: This paper focuses on the representation of climate change in French and Brazilian newspapers. It reports on the results of a discourse analysis of Le Monde, Le Figaro, O Estado de São Paulo and O Globo.The heuristic assumption driving this study is that the representation of climate change in the press is divided between utopic and dystopic ideas. The theoretical framework is based on philosophical works as well as on literary studies about utopia and dystopia. The concept of "moment discursif" and Sophie Moirand's works related to daily French press are the bases for discourse analysis. The study finds that the representation of climate change in the newspapers is shaped by the idea of scientific and politic consensus, promoting individual initiatives, and nature preservation and technologies as the answer for climate change.

Keywords: climate change; press; representation; utopia; dystopia; discourse analysis.

\section{Introduction}

Ce travail de recherche s'intéresse à la représentation du changement climatique dans les médias, et plus spécifiquement dans les presses quotidiennes nationales françaises et brésiliennes, au travers du prisme du discours. En tant que dispositif de représentation, la presse co-construit le sens et octroiedes pouvoirs aux acteurs qui participent à la construction du discours sur le changement climatique. En sélectionnant l'angle, les acteurs et les événements en relation avec le sujet, les médias permettent à certaines notions et idées de circuler, articulant ainsi un tissu de représentations qui participent à la construction sociale du risque climatique (Carvalho \& Burgess, 2005).

Cet article revisite les résultats de la recherche qui a été réalisée dans le cadre d'une thèse de doctorat sur la représentation du changement climatique dans des journaux brésiliens et français. La recherche se focalise sur la problématique de la représentation sociale d'un phénomène complexe d'ordre scientifique et politique. Au-delà de l'analyse de la médiatisation du changement climatique comme transmission d'un savoir scientifique, nous observons le phénomène comme une construction sociale et discursive, où opèrent les représentations des sociétés.

Nous partons des interrogations suivantes: comment le phénomène du changement climati- que est-il représenté dans la presse? Comment les rapports complexes entre sciences et politiques sont-ils représentés? En proposant une analyse comparative, nous nous demandons également si la construction de l'actualité sur le changement climatique et sa représentation se font de manière différente dans les deux pays.

L'hypothèse heuristique fil conducteur de l'étude est qu'il existe, dans les journaux analysés, une représentation qui se partage entre utopie et dystopie. Cette représentation propose parfois un retour aux valeurs des sociétés traditionnelles et non-industrialisées, parfois préconise au contraire des solutions technologiques faisant appel à l'idée d'une science salvatrice.

Pour notre analyse, nous nous sommes basés essentiellement sur les travaux de Sophie Moirand (2011) sur la presse quotidienne nationale. L'auteur puise ses sources dans les théories de Bakhtine, prenant en compte la dimension dialogique du discours, c'est-à-dire qu'il existe uniquement vis-à-vis d'autres discours antérieurs ou présents. Dans la conception dialogique du discours, tout énoncé a également un auteur et renvoie au moins à deux sujets, et donc à un dialogue potentiel (Todorov, 1981). Pour Bakhtine l'auditeur incarne le représentant accrédité, "qui participe activement à la parole intérieure et extérieure" de l'énonciateur (Bakhtine in Todorov, 1981, Ch. 5, par. 14). Cette relation éta- 
blie entre ces deux sujets est fondamentale dans la construction d'une théorie dialectique du discours, en insistant sur le rapport entre les pratiques sociales et le discours, en montrant le processus par lequel le discours est défini au travers des structures du langage, tandis qu'il les transforme et les reproduit (Chouliaraki \& Fairclough, 2001).

\section{La médiatisation du changement climatique en France et au Brésil}

Le risque climatique apparait dans la presse française aux alentours de 1987 (Rabeharisoa, 1997). Pendant les deux années suivantes, la production médiatique sur le sujet est faible. Mais les manifestations internationales qui se sont tenues entre 1989 et 1992, notamment lors de la Conférence des Nations Unies sur l'environnement et le développement à Rio de Janeiro, ont éveillé l'intérêt de la presse sur le sujet. Le travail que les médias ont entrepris collectivement pour se familiariser avec la question, entre également en compte pour une part non négligeable dans la couverture du risque climatique durant cette période. Mormont \& Dasnoy (1995) observent qu'à cette époque, en France comme en Belgique, l'environnement reste traité occasionnellement selon une approche tantôt consumériste, tantôt politique. L'absence d'émissions de télévision et de structures de production d'information stables s'intéressant au domaine de l'environnement se traduit par une faible spécialisation des journalistes. Vers la fin des années 1990, ce cadre change avec l'institutionnalisation de l'environnement comme spécialité journalistique et le changement climatique se trouve à être traité de façon récurrente dans la presse, d'une façon conforme aux logiques marchands des médias favorisant un traitement dépolitisé du sujet, sans remettre en question les logiques capitalistes à l'origine du problème environnemental (Comby, 2008).

La médiatisation du changement climatique en France a été analysée par différents auteurs, depuis une perspective discursive, sémiologique, ainsi que sociologique et sur différents aspects: la dramatisation et la construction de catastrophes conjuguées à la construction de la certitude et du consensus scientifique (D'Almeida \& Lins Peliz, 2011; Micheau, 2012; Bolka-Tabary, 2012), mais également les controverses, même si celles-ci ne sont pas très présentes dans le discours de la presse en France (Mauger-Parrat \& Lins Peliz, 2013), comme montre l'étude de Brossard et al. (2004) sur la couverture journalistique du changement climatique en France et aux États-Unis entre 1987 et 1997. Selon l'étude, les limites culturelles des pratiques journalistiques de chaque pays, font que certains sujets gagnent l'attention des médias pendant une certaine période ou pendant des cycles où l'attention donnée au sujet évolue (media attention cycles). Cette recherche montre une tendance des différenciations entre la presse française qui vise à ne pas donner de visibilité aux controverses scientifiques sur le climat, et la presse anglo-saxonne dont la médiatisation par la polémique et la controverse sur le phénomène nourrit les pages des journaux.

$\mathrm{Au}$ Brésil, la couverture des problèmes environnementaux et du changement climatique a beaucoup évolué dans les vingt dernières années et avec elle, l'intérêt des chercheurs. Accompagnant les tendances mondiales, le traitement des faits environnementaux a également pris du temps pour être considéré pertinent, ce genre d'événements émerge dans les médias vers la fin des années 1960 (Tourinho Girardi et al., 2012). La conférence des Nations Unies sur l'environnement et le développement à Rio de Janeiro, en 1992, a représenté également au Brésil, un jalon dans la couverture 
des problèmes environnementaux mais également $\mathrm{du}$ changement climatique dans la presse. À cette époque, les champs scientifique et politique se consolident en tant que voix légitimes pour le discours journalistique afin d'aborder les questions environnementales (Miranda Costa, 2006). Nous pouvons dire que c'est à partir des années 1990 que la problématique s'inscrit dans le discours de la presse. C'est toutefois dans la première décennie des années 2000 que le changement climatique s'installe de manière durable dans le discours de la presse brésilienne. L'année 2007 revêt plus d'importance, selon Miguel (2012), car dans cette période la couverture du sujet a connu un pic, avec la publication des rapports sur le changement climatique, qui a généré des discussions sur la nécessité de développer des carburants alternatifs aux fossiles, provocant le débat sur les biocarburants et l'éthanol.

Des analyses discursives de la construction de l'événement changement climatique dans la presse brésilienne révèlent une dominance d'articles descriptifs ou factuels, caractérisés par un ton d'alerte environnementale (Miranda Costa, 2006), ainsi que la prédominance des institutions gouvernementales, de recherche et universités en tant que source principale d'informations durant la périodeécoulée entre les années 1975 et les années 2000, malgré l'apparition des ONGs dans les années 1980 (Da Silva Delevati et al., 2013). Ainsi le discours scientifique est-il devenu la base du discours journalistique sur la thématique. Même si la science reste la source principale d'informations relevant du changement climatique dans la presse au Brésil, Da Silva Delevati et al. (2013) ont observé des conflits entre spécialistes et journalistes au Brésil, qu'elles attribuent au déséquilibre entre les valeurs de l'information et les contraintes de la routine professionnelle et l'épistémologie de la science. Des analyses sur les Conférences internationales COP 15 et COP 16, ont révéléla prédominance d'un discours technocratique: les acteurs des champs politique et économique semblent être privilégiés, au détriment des ONGs ou des représentants de la société civile (Tourinho Girardi, 2011).

Nous pouvons observer des points communs entre la publicisation du changement climatique dans les deux pays, marqués par des moments historiques semblables, avec des pics dans les années de grandes conférences mondiales. Cependant, il est difficile d'être catégorique, carsi l'approche de la médiatisation du changement climatique depuis un regard croisé a déjà intéressé différents chercheurs (Boykoff \& Roberts, 2007; Brossard et al., 2009; Painter \& Ashe, 2012) les analyses comparatives entre des pays émergents et développés sont pratiquement inexistantes. Les recherches sur la couverture des médias concernant les sujets environnementaux réalisées à un niveau international et comparatif entre pays, ouvre de nouvelles perspectives d'observation du problème du changement climatique, comme l'affirment Brossard et al. (2009). En ce sens, cet article peut apporter de nouvelles informations ou de nouvelles questions pour des études futures.

\section{Les concepts d'utopie et de dystopie au regard du changement climatique: vers un fondement analytique}

Les deux concepts structurants pour comprendre notre étude sont ceux d'utopie et de dystopie. Le premier évoque une idée de monde idéal, traité dans le livre de Thomas More, l'Utopie (1517). L'auteur anglais, inspiré par La République de Platon, tissait à l'aide de cet ouvrage une critique virulente contre la société anglaise sous le règne du roi Henri VIII, dans une période de guerres et de réformes reli- 
gieuses, à travers l'image d'une société, d'après lui, idéale. Le deuxième vocable, la dystopie, évoque le monde contre-idéal, une perspective opposée à l'utopie (Vieira, 2010). En effet, l'utopie n'existe pas sans la dystopie, la seconde représentant son côté sombre. En ce sens, les visions utopiques et dystopiques ne sont pas obligatoirement opposées. Une grande partie de l'histoire récente de la pensée utopique peut être lue comme un changement d'une force utopique vers une force dystopique (Booker, 1994).

Nous observons 1'utopie comme la construction d'imaginaires nécessaires pour l'être humain. La dimension utopique en tant qu'outil de la science nous intéresse. En ce sens, reprenant la pensée de Bachelard et Weber, Péquignot défend l'idée que l'utopie peut être considérée comme une méthode, et non uniquement comme un but ou une fin pour 1'action. "Il faut se projeter dans l'avenir pour décrire le chemin qu'on peut ou on veut prendre. Toute la question est d'accepter que ce "peut" ou ce "veut" ne se transforme pas en doit, sous peine de faire retomber la politique et la science dans la morale." (Péquignot, 1999, p. 107). L'utopie est alors un outil scientifique de prospection, dès que la tentation moraliste est évitée.

Cependant, nous croyons que quand l'utopie est utilisée au service de la science, elle présente plutôt les caractéristiques d'une dystopie, car c'est la menace du monde contre-idéalqui peut promouvoir l'analyse et le changement d'attitude. Ainsi, il est impossible d'échapper au fond moral, car son objectif est majoritairement culpabilisant.

L'idée de la présentation d'un futur sombre comme une feuille de route, est traité par Jean-Pierre Dupuy (2002) dans la perspective d'un “catastrophisme éclairé”, selon lequel le catastrophisme représente un événement impossible qui devient réalité. Une contradiction que Dupuy caractérise comme "à la fois catastrophiste et rationnelle." (Dupuy, 2002, p. 10) Dans le même sens qu'Hans Jonas (1998) et que son "principe responsabilité", Dupuy défend l'idée selon laquelle s'il faut prévenir la catastrophe, on a besoin de croire en sa possibilité avant qu'elle ne se produise. La catastrophe ne devient éventuelle qu'en se "possibilisant". Il est donc nécessaire de matérialiser la catastrophe pour éviter sa réalisation. L'auteur met en question le progrès et affirme qu'aujourd'hui "notre problème essentiel est d'éviter la catastrophe majeure." (Dupuy, 2005, Ch. 1, s.1, par. 10). En ce sens, celui qui n'est pas directement menacé ne se décide pas à réformer radicalement son mode de vie. L'absence de certitudes, compte tenu des connaissances scientifiques et techniques du moment, ne doit pas retarder l'adoption de mesures effectives et proportionnés, visant à prévenir un risque de dommages graves et irréversibles à l'environnent, ce à un coût économiquement acceptable. En présence d'une menace indirecte, l'homme réagit de façon indirecte, parfois rationnellement, parfois de manière irrationnelle. Mais pour Jonas (2000), les perspectives lointaines, notamment lorsque ce sont les générations à venir qui sont concernées, n'incitent manifestement pas les hommes à modifier leur comportement. En revanche, dès lors que la menace est directe, il en va autrement, tant sur le plan individuel que sur le plan collectif. "On ne prend la fuite que lorsque l'éruption volcanique s'est déjà déclenchée." (Jonas, 2000, p. 23). L'auteur met en avant l'idée du risque technologique par rapport à l'existence humaine, et défend le présupposé que pour envisager le risque dans une situation donnée, nous devons regarder la perspective la plus pessimiste.

Nonobstant, il existe un défi à relever pour que le catastrophisme soit éclairé: la nécessité de rendre crédible cette utopie-dystopie sans pour autant figer 
les actions. Il existe toujours le danger d'intimider le public avec des prévisions catastrophistes qui figeront l'action, en présentant des obstacles trop difficiles à surmonter. De cette façon, le résultat ne serait pas celui souhaité. La critique de ce principe est que la peur excessive génère une société de coercition, où les libertés individuelles ne sont pas respectées. Le catastrophisme éclairé porté à l'outrance se révèle le revers de l'attendu. Il ne peut pas être éclairé, il représente la dystopie. Dans les récits dystopiques, la peur génère une organisation sociale totalitaire, où la démocratie cède la place à l'aliénation. Le monde contrôlé empêcherait la société de délibérer et d'arriver à un point d'accord commun.

Dans le catastrophisme éclairé, ainsi que dans les utopies/dystopies il existe une part de réel et une autre d'imaginaire. Dans le premier cas cependant, les prévisions sont basées sur des modèles scientifiques de futur, c'est à dire que les technologies donnent une légitimité à ce futur imaginé.

Parmi les dystopies environnementales, le changement climatique est celle qui domine le plus le discours de l'actualité, soit par sa politisation, soit par son contenu dramatique. Le phénomène pointe du doigt notre manque de réserve morale, avec la rhétorique qui s'est popularisée sur les responsabilités individuelles et le développement du sens d'un "péché écologique" (Stableford, 2010, s.5, par. 3). Une des données les plus fortes dans l'observation du phénomène est la participation humaine, c'est-à-dire que l'humanité est la cause de ses propres malheurs et de sa propre destruction, idée qui contribue à cette dramatisation et à cette culpabilisation.

D’une façon générale, nous pouvons donc dire que le phénomène représente un exemple de dystopie. Mais nous croyons que le changement climatique et sa représentation sociale ont une part importante d'utopie, représentée par la science et l'utilisation massive des technologies très avancées qui ont permis de modéliser les climats du futur (Dahan, 2007), couplés aux scénarios d'émissions de gaz à effet de serre, qui permettent de faire des prévisions sur les climats futurs. Les scénarios visualisent les émissions des GES dans le futur, fondés sur des projections socio-économiques, tandis que les modèles projettent l'avenir du climat sur la planète. Guillemot (2007) soutient que les modèles numériques sont les seuls instruments capables de nous projeter vers l'avenir du climat de notre planète. Dans ce sens, les modèles numériques sont au centre de la question du changement climatique. C'est en s'appuyant sur les simulations faites avec ces modèles que des climatologues ont pu alerter le monde sur le déséquilibre du climat et s'organiser ensuite autour d'une expertise. Le travail d'expertise du climat est majoritairement basé sur ces modèles et sont utilisés comme sources pour les rapports du Groupe intergouvernemental d'experts sur l'évolution du climat (Giec), qui a son tour représentent les bases pour les négociations climatiques et pour la construction des politiques d'émission de GES.

Cependant, la modélisation n'échappe pas aux critiques. Des scientifiques des pays du Sud voient dans cette méthodologie, "un langage du Nord", comme 1'explique Dahan (2007, p. 19), qui efface le passé puisque fixant comme état initial l'année 1990, année du Protocole de Kyoto, et ne comptabilisant pas les années d'industrialisation et d'émission de GES des pays du Nord. Ce niveau additionnel serait, selon les experts, très difficile à évaluer. Cette méthode, selon Dahan, s'empare de processus globalisés, homogénéise des variables, annule les disparités locales. "Le choix de la température moyenne comme variable privilégiée accentue encore ce biais de globalisation." (Dahan, 2007, p. 20). 
Dans l'utopie de l'entente entre peuples, nous voyons l'imposition d'une pensée du Nord vers le Sud, génératrice de dystopie engageant les négociations et les décisions sur le climat. Le discours sur le changement climatique se base fortement sur la prévision scientifique élaborée avec l'utilisation de technologies, la compréhension du domaine spatial, ainsi que de la météorologie. C'est un monde rêvé, imaginé, parfois idéal, dans le cas des scenarios envisagés où la réduction de GES est atteinte, ou bien un monde effrayant, dans le cas où les prévisions plus catastrophistes se matérialisent. La science porte dans ce contexte une fonction légitimatrice de la vérité liée aux prospections; elle participe à la construction des imaginaires qui se posent dans un futur possible, une utopie résultant d'une dystopie initiale.

Ces "prévisions du futur" basées sur des modèles et scénarios échappent complètement à la compréhension du public non scientifique, elles appartiennent au monde technologique et sont faites par des ordinateurs, machines qui ont permis de construire une connaissance jamais observable sur le climat dans le monde jusque là. Mais le revers de cette utopie de la connaissance et du progrès humain est la peur de l'aliénation par la machine: nous ne comprenons pas le phénomène et dépendons de modèles pour l'interpréter. Ce fait génère de l'anxiété. Le climat, qui faisait partie d'une utopie d'équilibre, bien que non complétement réelle mais observable et interprétable par l'homme commun, passe dans une sphère technologique qui échappe au savoir commun.

\section{Une méthodologie de travail en trois dimensions}

Pour cette étude, un corpus formé par des articles issus de quatre quotidiens de grande cir- culation a été observé: $O$ Estado de São Paulo et O Globo, du Brésil, Le Monde et Le Figaro, de la France, entre les années de 2008 et 2012. Le choix des dattes correspond à la continuité d'un travail de recherche réalisé en Master en Recherche sur la représentation du changement climatique dans la presse française, qui a analysé les journaux entre les années 1990 et 2007. Le choix de quotidiens repose sur le croisement de différents critères de représentation de l'information sur le changement climatique comme espaces dédiés ou visibilité du sujet. Pour surmonter les difficultés posées par le cadre d'une analyse comparative, et le fait qu'au Brésil il n'existe pas de presse quotidienne nationale comme en France, nous avons choisi des représentants de deux états, Rio de Janeiro et São Paulo, avec une vocation de couverture des faits internationaux. Une recherche préalable dans les bases de presse Factiva et Europresse nous a montré que les journaux qui publiaient le plus d'articles avec les mots clefs changement climatique, mudanças climáticas, effet de serre, efeito estufa, réchauffement, aquecimento étaient Le Monde, Le Figaro, O Globo et $O$ Estado de São Paulo. Le choix d'analyser la presse provient de la caractéristique de ces médias à confronter et générer le débat, d'être un dispositif mettant en rapport des acteurs et générant des discours sociaux puissants.

La partie empirique du travail est composée de trois dimensions. La première de ces dimensions se concentre sur les macro-catégories du discours, relevant ainsi de l'observation globale de la "scénarisation médiatique d'un événement" (Moirand, 2011, p. 14), ou de l'ordre de la "superstructure" (Van Dijk, 1990). Il s'agit des structures globales du discours, définies par des catégories et règles spécifiques, qui participent de la construction de la cohérence locale des textes, elles sont régies par des stratégies cognitives partagées par l'auteur et le 
public (Van Dijk, 1990). Le système de rubricage, la mise en page, l'éditorial, entre autres, participent ainsi à la construction du discours, avec ses structures schématisantes et hiérarchisantes, et ont été pris en compte dans l'analyse. L'autre élément observé est le journaliste. En tant que médiateur cet acteur participe également de la mobilisation et de la construction d'un discours fondé sur plusieurs voix, contribuant à amplifier la ronde des dires ou permettant même sa matérialisation. À partir d'une analyse plus ténue des unités discursives, nous observons une articulation entre la superstructure $\mathrm{du}$ journal, les modes discursifs et les formes de description, de récit ou d'argumentation.

Dans une deuxième dimension de l'analyse, nous revenons au corpus, avec l'intérêt de délimiter les différents "moments discursifs" qui ont été repérés à partir d'une lecture diachronique du corpus. Pour l'analyse, nous avons amplement utilisé la notion de "moment discursif" (Moirand, 2011, p. 4), c'est-à-dire “des productions discursives qui surgissent, parfois brutalement, dans les médias, à propos d'un fait du monde réel qui devient par et dans les médias un "événement"" (Moirand, 2011, p. 4). Cette démarche nous a permis de mettre en lumière les événements dans une succession chronologique au travers de trois temporalités distinctes: le temps qui couvre l'évènement en question; le temps des évènements parallèles qui ont joué un rôle dans l'existence de l'événement principal; le temps du moment historique dans lequel l'évènement s'insère. Cette méthode ne consiste pas à hiérarchiser les contenus de la presse, sinon à montrer une séquence, à travers cette succession des vagues d'informations qui font émerger le sujet dans les pages des journaux. L'observation nous a permis de comprendre les choix de la presse analysée sur les sujets "d'actualité", et également les points communs et les points divergents entre la presse des deux pays analysés. À travers cette observation, nous construisons également un sous-corpus de moments discursifs analysables.

Après la structuration du corpus en sous-corpus, nous avons distingué les principales caractéristiques et les tendances du discours permettant de créer des catégories d'analyse concernant un niveau local des unités discursives, dans lequel les observables de l'analyse deviennent des "catégories qui renvoient aux fonctions essentielles du langage verbal: "représenter" et "communiquer"", et sur lesquelles s'appuie une analyse linguistique du discours (Moirand, 2011, p. 5). Cependant, l'intérêt de l'analyse n'est pas simplement de se rapprocher des événements à travers les "“"mots" qui les caractérisent et les constructions qui contribuent à les relier", dans un niveau syntagmatique, mais également sur la façon dont "le scripteur les distribue dans le déroulement linéaire du discours" (Moirand, 2011, p. 58), dans un niveau paradigmatique. En considérant la désignation des faits ou en se demandant comment on nomme, comment on désigne, comment on caractérise, comment on qualifie, comment on reformule.

Nous avons donc observé la circulation des notions (idées qui parfois finissent par devenir le nom de l'événement lui-même. "Copenhague" est devenu synonyme de la COP 15 par exemple), la nomination ou la façon dont les événements sont désignés. Ce qui nous intéressait, plus que d'observer les occurrences des mots figés dans un article précis d'actualité, c'était de révéler, dans un ordre chronologique et comparatif, l'évolution du nom en passant par une large palette d'émotions (de l'expectative à l'échec d'une conférence internationale, p.ex.), l'entourage, les allusions, etc. Nous avons également observé les acteurs et énonciateurs convoqués, ainsi que leur discours et la façon dont ils sont nommés, présentés ou introduits dans les 
textes des journaux et les rapports au temps par la présence matérielle des citations dans le texte ou des allusions.

Dans une troisième dimension, nous revenons finalement au corpus en nous posons la question suivante: comment les idées d'utopie et de dystopie se voient reflétées, s'opposent et se construisent dans le discours de presse analysée ? Alors, nous observons la façon dont les notions représentatives du phénomène émergent dans les journaux analysés, ainsi que les points en commun et ceux de divergences entre les discours de la presse brésilienne et de la presse française. L'observation d'éléments utopiques/dystopiques part du présupposé, qu'il n'existe pas dystopie sans utopie et vice-versa. Alors, les valeurs dystopiques doivent être présentées accompagnées de valeurs utopiques. Cette présence n'est pas affichée dans le texte, dans certains cas, mais se fait à travers l'allusion ou de ce que nous nommons les éléments extérieurs aux textes, évoqués par les auteurs à travers la supposition, en faisant appel à ce que Sophie Moirand appelle "mémoire discursive partagée". Cette mémoire participe à la construction de l'actualité, dans la mesure où les nouveaux sujets sont basés, de fait, sur un savoir partagé issu en grande partie d'une mémoire collective, selon la conception de M. Halbwachs, cité par l'auteur. La présence du lecteur est constante, elle définit une co-construction du discours.

\section{Les idées structurantes de la représentation du changement climatique}

Nous nous centrons dans les prochaines pages sur certaines idées forces structurantes d'une dichotomie utopie/ dystopie dans les journaux analysés: celle du consensus, qui s'oppose au dissensus ou au manque d'accord, les initiatives individuelles des citoyens comme un idéal utopique du travailler ensemble, le retour aux sources et les liens brisés entre nature et humanité, ainsi que la valorisation des technologies comme réponse au problème climatique.

\subsection{L'idée structurante du consensus}

La première idée force dans la représentation du changement climatique est celle du consensus qui structure le discours de la presse. Concernant la science, nous observons la construction, dans les pages des journaux, d'un consensus scientifique autour du changement climatique,tandis que dans le politique, nous observons la recherche d'un accordet la difficulté de l'attendre, dans les négociations internationales sur le climat. En ce sens, la représentation des oppositions se construit en grande partie autour de l'idée structurante du "consensus" qui régit les négociations sous l'égide des Nations Unies.

Le consensus politique est difficilement atteignable, que ce soit dans les conférences internationales, ou par les pouvoirs locaux au moment d'établir des règles pour pallier le changement climatique. Cette idée est observée à travers toute une sémantique qui évoque le processus de construction d'un accord, avec les débats et les objectifs en principe communs.

Ainsi, les notions sont diverses, nous observons des notions visant l'entente "accord", "traité", "texte commun". Nonobstant, les co-ocurrences de ces notions sont négatives, "échec", "fiasco", et évoquent la déception. Le journal OEstado de São Paulo publie quelques semaines avant la COP 15 un article sur les expectatives déposées sur les négociations qui ne sont pas élevées. Selon le texte, l'entente entre responsables des pays est brisée. 
Dans ce cas précis, il s'agit des décisions sur la continuité du Protocole de Kyoto, qui, comme nous le savons, n'ont pas été vraiment prises pendant la conférence de Copenhague.

Il existe l'idée, dans les journaux, de la nécessité que tous les pays "s'assoient" à la table des négociations et fixent des règles contraignantes pour diminuer les émissions de GES. Cette idée utopique est néanmoins brisée par le manque de perspectives d'un accord. Si nous observons de près les pays convoqués, nous ne pouvons pas dire que ces objectifs soient communs. Bien qu'il existe un objectif principal commun représenté par la protection du climat et la tentative d'éviter la montée des températures de la Terre, les pays présents dans chaque sommet préfèrent mettre en avant leurs intérêts politiques. Les causes sont en vérité individuelles. Les idées de conflit d'intérêt entre pays dépassent de beaucoup les enjeux du changement climatique. La transparence des processus politique empêche cette construction discursive, mais la recherche de cette idée est toujours présente, ainsi que celle d'un objectif commun entre pays si différents, voire opposés les uns aux autres.

\subsubsection{La représentation utopique des nouveaux} rapports de force qui cache des anciennes pratiques

La représentation de la difficulté d'arriver à un accord international trouve ses sources dans l'opposition des acteurs participant à ces réunions, qui se disputent dans le terrain discursif des journaux. En ce sens, et selon le journaliste Hervé Kempf du Monde, la COP 14 réalisée à Poznan est "une épreuve de force entre 1'Union Européenne et les pays du Sud", où, "Finalement, ils (les pays du Sud) l'ont remportée...". La position géographique sem- ble avoir un poids important dans la dénomination des acteurs. Parler des "pays du Sud" est un choix moins engageant que de créer une dichotomie entre les "pays industrialisés" et "non industrialisés", dénomination qui ferait reposer les responsabilités des émissions de GES sur les "pays développés", non sur les "pays en voie de développement". Cette terminologie représente la face onusienne des négociations, qui efface les différences de développement. Néanmoins, Le Monde parle des pays du "Sud" sans parler des pays "du Nord". Le choix est de parler d'Union Européenne. Les intérêts de la France ne sont pas cités. L'hétérogénéité des idées entre ces pays "du Sud" et les pays européens est effacée au détriment de la construction de deux blocs qui s'opposent.

Pour sa part, le correspondant du Figaro à Bruxelles, Jean Jacques Mevel représente la COP 15 comme un théâtre des disputes politiques, cette fois-ci entre la Chine, les États-Unis et l'Union Européenne.

\begin{abstract}
À Copenhague, l'ambition se frotte aux réalités. D'ici à Noël, l'Europe saura si elle a bien fait d'investir son crédit - mais peu d'argent - dans un pari auquel ni les États-Unis ni la Chine ne sont encore prêts à souscrire [...] L'Union Européenne joue gros. Pour une puissance qui rêve de compenser dans l'art de la persuasion (soft power) ce qui lui manque de muscle et de cohésion, le pire serait une espérance déçue. (Jean Jacques Mevel, Le Figaro, Opinions, 15/12/2009)
\end{abstract}

Dans un autre article du même journal, le journaliste affirme que les débats à Copenhague mettent "le leadership européen à rude épreuve". Nous n'observons aucune fois, dans l'extrait, le mot changement climatique. On parle d'ambitions, d'imposer un leadership sur le sujet, d'investissement ("investir son crédit"), du "manque de muscle et de cohésion", on fait une critique à propos de la 
stratégie de l'Europe. Nous observons dans cette mise en scène que les enjeux ne sont pas simplement liés à la réduction des émissions de GES à propos du changement climatique, il s'agit bien d'une lutte qui reflète des positions géopolitiques plus importantes et plus fortes, synthétisées par la construction “L'Europe joue gros". L'Europe joue en vérité le leadership des pays industrialisés face aux exigences croissantes des pays en voie de développement et émergents, ainsi que la place de puissance mondiale. Les débats sur le changement climatique sont donc de nouvelles zones d'influence pour les pays industrialisés afin de réaffirmer leur hégémonie. Ils se heurtent cependant une certaine insoumission.

Dans ce champ de gouvernance internationale, nous observons toutefois une restructuration des acteurs, où de nouveaux leaderships semblent se construire, en même temps qu'ils sont questionnés.

Très applaudi le matin, Lula a promis de l'argent pour le fonds climatique et a révélé sa frustration lors des négociations ${ }^{1}$. (Lisandra Paraguassu, O Estado de São Paulo, Vida\&, 19/12/2009)

L'objectif, auquel le Brésil et les pays du G77 - les "en développement" - s'opposaient, a été presque atteint. ${ }^{2}$ (Andrei Netto, Afra Balazina, O Estado de São Paulo, Vida\&, 21/12/2009).

Parmi les morts et les blessés, celui qui sort avec l'image la plus abîmée est le président américain, Barack Obama, qui est sorti d'une réunion avec la Chine, l'Inde, l'Afrique du Sud et le Brésil en annonçant être arrivé à un accord sans précédent avec les pays émergents [...] Obama n'a permis aucun accord. Ce qu'il a fait a été de taper du pied pour convaincre les autres pays - Brésil inclus - que c'était mieux d'avoir un accord faible (mais qui correspondait aux intérêts des États-Unis) qu'aucun accord. Il a fini sans aucun accord et pourra encore dire que ce sont les pays pauvres qui, en refusant l'accord, ont provoqué l'échec de la conférence ${ }^{3}$. (Herton Escobar, Un échec "sans précedents", O Estado de São Paulo, Vida\&. 20/12/2009).

Dans les extraits du journal O Estado de São Paulo sur la COP 15, nous observons la construction du Brésil dans les négociations comme un pays dont les idées du président sont bien accueillies ("très applaudi"), il se montre capable de réorganiser la disposition des pays autour de son leadership et faire barrage aux idées contraires. Il s'agit cependant d'un blocage peu solide, car l'objectif de l'opposition a été "presque" atteint. L'adverbe semble mettre en question le leadership brésilien et la force des pays "en développement". Ce nouveau leadership est encore mis en question par le troisième article du même journal. D'après le texte de l'article du Estado de São Paulo, écrit par le journaliste Hector Escobar, le président américain Barack Obama, "n'a permis aucun accord", "il a tapé du pied" pour défendre ses intérêts. Le verbe "permettre" montre qu'il existe un pouvoir de décision, qu'il y a bien des pays qui décident. Le journaliste décrit les faits comme une stratégie d'Obama permettant de faire

\footnotetext{
${ }^{1}$ Libre traduction. Version originale en portugais:"Muito aplaudido pela manhã, Lula prometeu dinheiro para fundo climático e revelou frustração com negociações" (O Estado de São Paulo, Lisandra Paraguassu, Vida\&, 19/12/2009).

${ }^{2}$ Libre traduction. Version originale en portugais: "O objetivo, ao qual Brasil e os países do G77 - os "em desenvolvimento" - se opunham, quase foi alcançado.” (Andrei Netto, Afra Balazina, O Estado de São Paulo, Vida\&, 21/12/2009).

${ }^{3}$ Libre traduction. Version originale en portugais: "Entre mortos e feridos, quem sai com a imagem mais arranhada é o presidente americano, Barack Obama, que saiu de uma reunião com China, Índia, África do Sul e Brasil anunciando ter fechado um acordo "sem precedentes" com os países emergentes [...] Obama não intermediou acordo nenhum. O que ele fez foi bater o pé e convencer os outros países - Brasil incluído - que era melhor ter um acordo fraco (mas que atendia aos interesses dos EUA) do que acordo nenhum. Acabou sem acordo nenhum, e ainda poderá dizer que foram os países pobres, ao rejeitar o acordo, que causaram o fracasso da conferência." (Herton Escobar, Um fracasso 'sem precedentes', O Estado de São Paulo, Vida\&, 20/12/2009).
} 
peser la responsabilité d'un "échec" du sommet de Copenhague sur les “pays pauvres”. L'idée qui émerge de l'extrait est que, bien que le consensus entre tous les pays est toujours rappelé comme base des négociations onusiennes, les rapports de force dans les négociations onusiennes maintient un statuquo de la géopolitique mondiale, dans laquelle les pays les plus riches ont le pouvoir de décision.

La recherche d'un consensus politique est reprise dans la presse et restructurée de façon à construire des nouveaux rapports de force, en rétablissant une nouvelle géopolitique mondiale, où des régions ou pays sans voix gagnent force. Ces rapports se construisent de manière différente dans les journaux brésiliens et les journaux français analysés. Nous observons donc que la presse française se centre sur une perspective utopique des nouvelles gouvernances climatiques, tandis que la presse brésilienne met en question cette réorganisation. Les efforts de la France en tant que pays individuel ne sont pas mis en avant, la perspective est européenne, l'objectif étant de se maintenir dans sa principale fonction de leader et dicter les règles de la situation. L'ancrage de cette construction est l'idée du consensus extérieur au texte, mais structurant dans le sens qu'il construit un discours autour du changement climatique dans la presse, dans des scénarios d'apparents nouveaux rapports de forces mais qui revalident d'anciennes pratiques politiques au sein de l'ONU.

\subsubsection{Le consensus scientifique contre le manque d'accord politique}

Nous observons que le discours scientifique est représenté dans les journaux de manière consensuelle, ou qu'un consensus scientifique est construit dans les pages des journaux. La construction d'un consensus scientifique est nécessaire car de cette construction émane une opposition structurante: le discours de la presse repose d'une façon implicite sur l'idée d'un consensus scientifique qui s'oppose fortement à celle du manque d'accord parmi les acteurs politiques. Cette réalité est observée plus fortement lors des conférences internationales sur le climat.

[...] toutes les recherches récentes indiquent que les changements climatiques sont beaucoup plus rapides de ce qu'on imaginait quelques années auparavant ${ }^{4}$ (Herton Escobar, Un échec sans précédents, O Estado de São Paulo, Vida\&, 20/12/2009)

Le phénomène vient d'être confirmé par une étude du Programme des Nations Unies pour l'environnement (PNUE)... (Le Monde, 17/11/2008)

Au travail des hommes politiques impliqués dans les conférences, nous observons donc l'opposition du travail des scientifiques. Du côté de la science, où nous voyons l'émergence de verbes qui indiquent la certitude, conjugués au présent donnant l'idée parfois de l'ordre, parfois de l'action. Les scientifiques disent que le monde "doit" réduire les émissions, les recherches "indiquent”, la modalité déontique qualifie les discours scientifiques présents dans la presse, les scientifiques "indiquent", "affirment". La généralisation au travers d'expressions comme "toutes les recherches" aide à construire l'idée d'un consensus scientifique. Le Monde vient même à affirmer que "le phénomène vient d'être confirmé par les Nations Unies, à travers le PNUE", alors que le PNUE n'est pas une autorité scientifique. Du côté politique, nous observons la déception: après " 15 jours de négociations" et "les

\footnotetext{
${ }^{4}$ Libre traduction. Version originale en portugais:“[...] todas as pesquisas recentes indicam que as mudanças climáticas estão ocorrendo muito mais rápido do que se imaginava até poucos anos atrás” (Herton Escobar, O Estado de São Paulo, Vida\&. Um fracasso 'sem precedentes', 20/12/2009).
} 
alertes des scientifiques", des objectifs de réduction d'émission de gaz à effet de serre n'ont pas été fixés.

Cette opposition ne se fait pas dans les espaces des Conférences à proprement parler, elle se fait dans l'interdiscursivité (Bakhtine, 1970) du discours des journaux, où plusieurs discours se rencontrent, dans l'exposition des recherches qui montrent que, d'un côté, il existe un consensus entre les hommes de science et de l'autre côté, on repère un manque de consensus du monde politique. L'échec est observé donc du côté des hommes politiques qui peinent à se mettre d'accord, ce qui n'est pas le cas pour la science.

L'idée qui structure la pensée de la presse brésilienne comme française est résumée par la journaliste Laurence Caramel, du Monde: "En clair, les scientifiques ont fait leur travail. Aux politiques maintenant de faire le leur". La science est alors source de consensus, tandis que la politique représente le désaccord.

\subsection{Des initiatives individuelles des citoyens et la dépolitisation des enjeux}

Dans le corpus analysé, certains articles sont dédiés à des initiatives menées par la société civile pour "lutter contre" le changement climatique. Ces actions ont été observées principalement dans les journaux brésiliens dans lesquels une action prend une place importante, celle de l'heure de la planète, 60 minutes pendant lesquelles un grand nombre de capitales éteint les lumières des points touristiques, de façon à inciter les personnes à suivre l'exemple. Cet événement, très visuel, porte un caractère symbolique fort, et se répète chaque année étudiée. À chaque fois, l'opération événementielle permet la construction de nouveaux articles dans la presse analysée.

\begin{abstract}
À l'étranger, les manifestations d'alpinistes dans le sommet de l'Himalaya et des plongeurs dans la Grande Barrière de Corail en Australie ont lieu. En Hongrie, des baigneurs iront sauter dans les bains publics de Budapest, pour une performance synchronisée. Au Népal, des jeunes et des moines irons marcher jusqu'au temple de Swayambhunath et former le numéro 350 avec des lanternes traditionnelles ${ }^{5}$. (Afra Balazina, Le jour d'action pour le climat rassemble de événements créatifs, O Estado de São Paulo, 24/10/2009).
\end{abstract}

Dans l'extrait, nous observons une séquence d'activités dans des lieux très lointains tel que l'Himalaya et la Grande Barrière de Corail, tous très représentatifs des espaces naturels qui subiront les effets du changement climatique, et qui font partie des imaginaires de la société moderne concernant les lieux à préserver. On y perçoit un certain exotisme:"des moines" qui marcheront jusqu'un temple, et un retour au traditionnel:"des lanternes traditionnelles." En plus de l'effet symbolique, l'idée de la construction d'un effort globale et commun pour éviter le changement climatique est ici renforcée, tout comme l'idée que chaque personne peut intervenir. L'objectif est de "réduire les effets du CC" et que le public peut participer à cette réduction d'une manière "créative", ainsi que l'affirme le titre de l'article. Bien qu'il ne soit pas clairement expliqué la façon dont ces actions peuvent intervenir dans la réduction des émissions de GES, sinon pour attirer l'attention sur le problème.

\footnotetext{
${ }^{5}$ Libre traduction. Version originale en portugais: "No exterior, destacam-se as manifestações de alpinistas no alto do Himalaia e de mergulhadores na Grande Barreira de Corais da Austrália. Na Hungria, banhistas vão saltar nos banhos públicos em Budapeste para uma performance sincronizada. No Nepal, jovens e monges vão marchar até ao templo de Swayambhunath e formar o número 350 com lanternas tradicionais." (Afra Balazina, Dia de ação pelo clima reúne eventos criativos. O Estado de São Paulo, 24/10/2009).
} 
Dans $O$ Globo, nous observons des manifestations similaires.

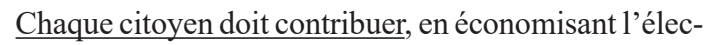
tricité, en plantant des arbres, en recyclant les déchets et en réglant le moteur de leur voitures. La planète est en train d'être détruite, dû à la cupidité, au productivisme, au gaspillage et à l'irresponsabilité. Nous devrons agir de manière solidaire, planifiée et globale, avant que la fonte des glaciers inonde villes et pays, détruise les espèces qui survivent aux pratiques prédatrices. ${ }^{6}$ (Carlos Minc, ministre de l'environnement, "Avant l'inondation...", O Globo, 30/05/2009)

Le contrôle d'émission des polluants et la création d'un fonds pour la compensation environnementale, l'usage d'énergies renouvelables à l'industrie et le ramassage de l'huile utilisée dans la cuisine des écoles publiques. Les solutions, qui représentent un cout zéro pour les coffres publics et combattent le réchauffement global, ont été les trois idées les plus votées, hier, dans la clôture du IVe Parlement des jeunes. Les projets de loi seront adressés pour analyse au gouverneur Sérgio Cabral, et peuvent être proposés au vote à l'Assemblée Législative. La démarche, réalisée par l'Alerj (Assemblée Législative de l'État de Rio de Janeiro) conjointement au secrétaire de l'Éducation de l'État de Rio, a amené 40 élèves des écoles publiques à connaitre le fonctionnement de l'Assemblée Législative et à proposer, pour la première fois, des projets de combat contre le réchauffement global. ${ }^{7}$ (Soares, Natália. "Les élèves suggèrent des actions de combat au réchauffement”, Rio, O Globo, 13/12/2008)

Dans le premier extrait, l'idée part de l'individuel, "chaque citoyen doit contribuer" avec des actions du quotidien comme économiser l'énergie, planter des arbres, recycler les déchets, pour arriver au global, à la protection du climat et la préservation de la planète, sans passer par le niveau local des pouvoirs publics. C'est la somme d'une multitude d'actions individuelles. Ce premier texte est d'autant plus représentatif car il est signé par le Ministre de l'Environnement de l'époque, Carlos Minc. La "cupidité", le "productivisme" n'ont pas de source dans le texte du Ministre, ainsi que "l'irresponsabilité". Il manque un sujet porteur de ces caractéristiques. Cette information doit être complétée par le lecteur, qui dira si ce sont les gouvernements ou les grandes organisations ou, encore, les citoyens. Indépendamment des responsables, l'action doit partir de ces derniers, à travers la reforestation ou l'économie d'électricité. Les instances gouvernementales sont, de cette façon, déresponsabilisées.

Dans le deuxième extrait, ce sont des enfants ou les nouvelles générations qui proposent des actions qui passeront possiblement par des instances politiques postérieurement. Ce fait n'est pas anodin, car les enfants représentent le futur déjà compromis par les actions du présent. De plus, les actions proposées ne représentent aucune dépense pour l'État, ou un "coût zéro pour les coffres publics". En même temps, cela apporte une charge de promotion pour les instances politiques: cela signifie que l'Assemblée de l'État de Rio s'ouvre aux idées des jeunes citoyens. Les idées sur le réchauffement émergent

\footnotetext{
${ }^{6}$ Libre traduction. Version originale en portugais: "Cada cidadão deve contribuir, economizando energia, plantando árvores, reciclando lixo e regulando o motor de seus carros. O planeta está sendo destruído, devido à ganância, ao produtivismo, ao desperdício e à irresponsabilidade. Devemos agir de forma solidária, planejada e global, antes que o derretimento das geleiras inunde cidades e países, destruindo as espécies que sobreviveram às práticas predatórias." (Carlos Minc, "Antes da Inundação...”, O Globo, 30/05/2009).

${ }^{7}$ Libre traduction. Version originale en portugais:"Controle de emissão de poluentes e a criação de um fundo para compensação ambiental, uso de energias renováveis na indústria e coleta de óleo de cozinha nas escolas públicas. As soluções, que representam custo zero para os cofres públicos e combatem o aquecimento global, foram as três idéias mais votadas, ontem, no encerramento do IV Parlamento Juvenil. Os projetos de lei serão encaminhados para apreciação do governador Sérgio Cabral, e podem voltar para a votação na Assembléia Legislativa. A iniciativa, realizada pela Alerj em conjunto com a Secretaria estadual de Educação, levou 40 estudantes da rede de ensino público para conhecer o funcionamento da Assembléia Legislativa e propor, pela primeira vez, projetos de combate ao aquecimento global."(Soares, Natália. "Alunos sugerem ações de combate ao aquecimento", Rio, O Globo, 13/12/2008)
} 
de la société, des jeunes citoyens. L'État ne semble pas intéressé pour faire de grandes dépenses dans ce projet.

L'idéologie qui émerge du discours est celle de la désinstitutionalisation à travers la promotion d'initiatives individuelles ou de la société civile. Les deux extraits mettent en scène une sorte d'approximation des institutions, qui sont du même coup désengagées de leur responsabilité: en lançant un appel à la participation des citoyens ou en s'ouvrant à des propositions d'élèves, le Ministre de l'Environnement et l'Assemblée de Rio donne la responsabilité des actions pour pallier le changement climatique aux citoyens; dans le même temps les instances politiques reconnaissent leur incapacité ou leur désengagement pour agir sur le sujet. C'est l'utopie de l'agir ensemble qui apparaît contre la dystopie des instances politiques.

\subsection{Les liens brisés et l'ideal utopique de preservation}

Dans nos analyses, nous avons également observé un groupe d'articles qui fonctionnent selon la logique de la représentation de la nature en tant que sanctuaires, où l'idée de préservation est forte. Dans ce discours, trois idées se croisent: celle des espaces naturels idéalisés comme témoins de l'équilibre de la terre, qui se joint à la deuxième idée, celle d'un retour aux sources du passé, et la troisième au maintien et la protection de la vie sur la Terre. Ces idées semblent entrer en conflit avec les valeurs de la société moderne.

Dans les articles qui évoquent ou parlent directement du changement climatique dans les journaux analysés, nous observons que l'espace naturel fait partie des textes, il existe en tant que réceptacle pacifique et instance victime des actions de l'homme. Cet espace naturel est le résultat d'une construction symbolique basée sur son idéalisation, et sur l'idée dystopique que leur déséquilibre peut être incontrôlable et méconnu.Nous arrivons à cette observation car les idées de "perte de contrôle"et de "point sans retour" sont évoquées dans les textes analysés, en relation à la perte de la forêt tropicale, par exemple. Nous observons également l'opposition entre un "équilibre actuel" et un équilibre "nouveau, imprévisible" et "l'incertitude". L'homme va en direction de l'inconnu, lequel semble être pire que les prévisions négatives mais déjà vécues, comme les sécheresses ou inondations, études scientifiques à l'appui. Ainsi, nous observons toute une construction de l'incertain. L'incertitude sur le futur de ces endroits dénote également un manque de connaissances empiriques à leur égard, normalement méconnues du grand public, sauf au travers de mythologies construites autour de ces lieux symboliques. Ils n'ont pas été touchés par l'homme, et il existe une croyance partagée par l'auteur du texte et son public que le maintien de ces endroits est important pour l'équilibre de la vie sur la Terre.

La Forêt Amazonienne, les sommets des Alpes, le Kilimandjaro ou les points extrêmes de la planète, Arctique et Antarctique, sont ce que nous appelons des lieux idéologiques construits. Car ils sont le résultat de constructions utopiques autour du paysage naturel, en même temps qu'ils fonctionnent comme garants, vigilants ou thermomètres d'un équilibre sur la terre. Nous observons ce rôle de ces espaces naturels évoqués également dans les journaux français: "Dans la glace des pôles se cache l'histoire de notre climat" ou "quand les pôles jouent à la bascule" (Le Monde, 2011) des titres et intertitres du Monde.

Ces espaces sont connus des lecteurs au travers des récits racontés par des émissions télévisés sur la 
nature, des films ou par des textes des journaux. La presse fonctionne alors comme un médiateur entre la société urbaine occidentale et l'environnement naturel, en construisant une représentation de ces espaces.

Les images idéologisées ne se réfèrent pas seulement aux lieux, elles vont plus loin, et font appel aux images romantiques du passé et des traditions, comme dans l'article du Monde.

La petite île de Nguna, dans l'archipel du Vanuatu (à $1750 \mathrm{~km}$ à l'est de l'Australie), est sur le point d'inaugurer son nouveau nakamal. Ce grand bâtiment, situé à quelques dizaines de mètres de la plage, ressemble à une coque de bateau renversée, tout en bois [...] Alors que, depuis des décennies, le nakamal était construit en utilisant du ciment et de l'acier, matériaux introduits par les missionnaires, le village a décidé de revenir au mode de construction traditionnel, jugé plus efficace pour résister aux vents violents. (Marie-Morgane Le Moël, Le Vanuatu veut anticiper le dérèglement du climat. Planète, LeMonde, 31/01/2011)

Pour lutter contre le changement climatique, les habitants de l'île cherchent dans l'architecture traditionnelle une meilleure protection contre les intempéries futures. Les matériaux utilisés dans la construction et qui ne sont plus assez solides, comme le ciment et l'acier, ont été introduits par les occidentaux.

Le rétablissement des cultures traditionnelles serait donc une manière de reconstituer ce lien brisé entre l'humanité et la nature, qui se concrétise par un retour à la Genèse, au moment de la conception du monde, où les sociétés vivent encore de manières traditionnelles.

Nous observons donc un équilibre brisé. Le changement climatique est donc le problème, le signe du fait que l'homme est arrivé dans ces endroits, non de façon directe mais à travers ses actions, les émissions de gaz à effet de serre qui contribuent au réchauffement climatique en causant le dégel dans les pôles, ou la déforestation dans le cas de la forêt tropicale. Ces sanctuaires qui servaient de thermomètres de l'équilibre de la vie sur la terre sont aujourd'hui menacés. L'utopie devient dystopie à travers cette présence non désirée de l'homme. La représentation de la nature et du milieu naturel dans la presse analysée est dichotomique.

Cette séparation est renforcée par l'idée de pureté dans la représentation des lieux naturels dans les articles. "Immaculée" est le mot utilisé par le journaliste du Monde pour définir le Groenland. Par ailleurs, l'homme est arrivé à cet endroit. Le Kilimandjaro, l'Himalaya, tout comme la forêt Amazonienne, les continents gelés, ces endroits mythiques, fonctionnent comme des lieux idéologiques construits; les lecteurs n'ont pas été dans ces endroits, néanmoins, tous se sont sentis concernés par la fonte des neiges dans ces montagnes. Les articles ont un ancrage dans une croyance partagée que de tels endroits doivent être préservés, ils gardent les mystères de la Terre et représentent les dernières vestiges d'une vie sauvage, de la nature, des paysages utopiques, d'une idée du passé, d'une autre manière de subsistance. Car le changement climatique met en question les rapports de l'homme et de la nature, et en ce sens, ces endroits témoignent qu'il reste encore une espérance pour la vie sur la planète.

\subsection{La géo-ingénierie et la science salvatrice}

Le sauvetage de la planète et le combat contre le changement climatique exigent de nouvelles armes:davantage d'énergies propres, des voitures plus performantes et moins contaminantes, des maisons plus efficaces et moins "énergivores". Ces 
idées sont évoquées dans le discours de la presse analysée comme des solutions venues d'un futur proche et prometteur. Elles sont définies comme géo-ingénierie, c'est-à-dire issuesde l'intervention délibérée et à grande échelle sur le système climatique, afin de contrer le réchauffement de la planète ou d'en atténuer certains effets. Elle est généralement divisée en deux grandes catégories: les techniques de capture du carbone visant à extraire l'excès de $\mathrm{CO} 2$ de l'atmosphère, et à le stocker dans un endroit où il est moins dangereux; et les techniques de gestion du rayonnement solaire (Hamilton, 2013). Présentée comme une voie d'échappement au cas où les mesures proposées ne marchent pas ou ne soient pas mise en pratique, sa force réside dans l'idée de trouver une réponse au changement climatique dans le "projet moderniste de domination de la nature par des moyens techniques." (Hamilton, 2013: chap.5, parr.1), de dominer les aléas du climat.

Cette notion intéresse spécialement deux journaux analysés, Le Figaro et $O$ Globo. Nous pouvons alors dire qu'il existe une marchandisation du sujet, par la présentation du progrès technologique comme une marchandise commercialisable. Cependant l'idée d'intérêts de la participation des entreprises et des États reste discrète dans les textes. L'idée utopique est portée par la décrédibilisation des opposantsaux technologies et des éléments dystopiques, ainsi que par la confiance dans la science, qui légitime le discours des acteurs experts défenseurs des solutions techniques.

Comme nous l'avons dit auparavant, le changement climatique représente le lien brisé entre l'homme et la nature, touchant même aux endroits considérés sacrés dans notre culture, protégés de la présence humaine. Pour reconstruire le lien, l'homme cherche donc un retour aux sources, soit culturel (la construction des maisons de façon tradi- tionnelle), soit technologique (la recherche d'énergies moins contaminantes). Cependant, avec la géo-ingénierie nous passons d'une idée romantique de préservation et de reconstruction du lien brisé, au retour à l'ancien rêve utopique de domination du climat et de la nature, qui amène avec lui la dystopie.

Cette représentation n'est pas, à notre avis, conflictuelle, car elle repose en grande partie, sur une idée de dépolitisation du problème, c'est-à-dire un effacement du débat politique à travers un détournement de l'attention des causes structurelles, pour la porter sur des aspects techniques, marchands ou moraux (Comby, 2015).

En ce sens, la science joue un rôle important. La science et la technologique, dans le discours des journaux, présentent deux côtés, un utopique et un autre dystopique, en étant à la fois la cause et la solution du problème. Elle est présentée comme la solution auxproblèmes causés par le changement climatique, ainsi qu'une des manières principales de mitigation du phénomène.

\section{Considérations finales}

L'hypothèse heuristique ayantguidée ce travail nous a amené à observer l'opposition des idées utopiques et dystopiques dans les quatre journaux analysés. Nous pouvons dire que les représentations utopiques sont liées tantôt à l'idéal du consensus structurant de la représentation de la gouvernance et de l'expertise du climat, tantôt à la représentation des espaces naturels. Cependant nous observons que le schéma utopique/dystopique se manifeste systématiquement dans la représentation du manque d'accord politique. L'expertise est toujours préalable aux décisions politiques, elle se réalise de façon indépendante, selon une représentation linéaire de l'expertise qui fonctionne en tant que garante de 
la vérité du processus politique. Les observations scientifiques fonctionnent comme des arguments d'autorité pour la prise des décisions politiques internationales.

Nous observons également qu'une idée utopique d'une nouvelle organisation géopolitique des leaderships est construite, ou des pays plus faibles semblent s'imposer au plus puissants. Cette représentation est particulièrement marquée dans $L e$ Monde. Mais cette vision ne se soutient pas dans le cadre de l'analyse comparative, car nous observons un regard plus critique de la part de la presse brésilienne sur le rôle du Brésil dans les grandes conférences internationales, principalement dans le cas du journal $O$ Estado de São Paulo. Si nous comparons ces deux représentations, il émerge alors une représentation dystopique des négociations qui perpétuent d'anciennes pratiques politiques, du côté de la presse brésilienne, et une vision utopique d'un nouvel ordre mondial du côté de la presse française. Il reste cependant complexe de comprendre les raisons de cette représentation, nous songeons à l'expression des idéologies et des positions politiques des journaux en question, transparaissant dans la représentation d'un événement international. Point intéressant à exploiter dans de futures études.

Le discours des journaux analysés se trouve également proche des idées de préservation de la nature et des derniers lieux idéalisés qui, selon ce discours, n'ont pas été touchés par l'action humaine. Le changement climatique symbolise le lien brisé entre l'homme et ces espaces naturels idéalisés. Le retour aux sources traditionnelles ainsi qu'à la nature, agit pour rétablir ces liens. En même temps, la technologie est présentée comme salvatrice à travers les solutions proposées au travers de la géo-ingénierie, particulièrement dans les journaux $O$ Globo et Le Figaro. Nous observons donc un schéma utopique/dystopique complexe où le lien entre homme/nature est reconstruit, soit au travers d'un retouraux sociétés traditionnelles et à la nature primitive, soit au travers des solutions technologiques. Nous croyons que ces deux représentations qui semblent en principe opposées, partagent les pages des journaux, car elles reposent sur l'idée de dépolitisation du changement climatique.

Nous considérons que la représentation utopique/dystopique est née en grand partie d'un regard dichotomique de la pressesur le changement climatique: entre la nature et l'homme, entre la science et le politique. De la nature et l'homme, nous observons donc la naissance d'un discours où il existerait des endroits préservés de la présence humaine, sans considérer que tout espace naturel est le résultat d'une construction symbolique liée aux imaginaires humains. De la dichotomie science et politique, nous observons un effort pour présenter la science comme représentante du réel, de la vérité, et également de l'achevé, tandis que le politique est le chaos, le désaccord et en processus. La science est neutre et protégée de la pensée subjective du politique. Cependant, un certain nombre de points reste à exploiter, tel que le poids des idéologies qui dominent la production de l'information dans cette représentation dichotomique, ainsi que le poids des institutions internationales comme l'ONU dans la construction du discours des journaux sur le changement climatique. Cette étude n'avait pas l'objectif de répondre à toutes les questions posées sur la représentation du changement climatique, sinon de proposer de nouvelles pistes sur les problématiques liées à la médiatisation du phénomène. 


\section{Références}

Bakhtine, M. La poétique de Dostoïevski. Paris: Seuil, 1970.

Bolka-Tabary, L. Le changement climatique à la télévision: de la science à la fiction, Communication et Langages, 172, 53-67, 2012. doi: 10.4074/S0336150012002049

Booker, K. The dystopian impulse in modern literature: fiction as social criticism. Connecticut: Greenwood, 1994.

Boykoff, M.; Roberts, T. Climate change and human development - risk and vulnerability in a warming world: Media coverage of climate change - current trends, strengths and weaknesses. United Nations Development Program Human Development Reports, 2007.

Brossard, D.; Shanahan, J.; McCommas, K. Are issue-cycles culturally constructed? A comparison of French and American coverage of global climate change. Mass Communication and Society, 7(3), 359-377, 2009.

Carvalho, A.; Burgess, J. Cultural circuits of climate change in U.K. broadsheet newspapers 1985-2003, Risk Analysis, 25(6), 1457-1469, 2005. doi:10.1111/j.15396924.2005.00692.x

Chouliaraki, L.; Fairclough, N. Discourse in late modernity. Rethinking critical discourse analysis. Edinburgh: Edinburgh University Press, 2001.

Comby, J.-B. Créer un climat favorable. Les enjeux liés aux changements climatiques: valorisation publique, médiatisation et appropriations au quotidien. Thèse (Doctorat en Sciences del'information et de la communication) - Institut Français de Presse et Université Paris 2, 2008.

Comby, J.-B. La question climatique. Genèse et dépolitisation d'un problème public. Paris: Raisons d'Agir, 2015.

Dahan, A. Modèles et fabrications du futur: du débat sur la croissance au débat climatique et retour. In: Dahan, A. Les modèles du futur. Paris: La Découverte "Recherches", 2007. p. 7-18.

Da Silva Delevati, A.; Franz Amaral, M. Miradas cruzadas: os campos científico e jornalístico na cobertura dos desastres climáticos. Revista Interamericana de Comunicação Midiática, 12(23), 20-38, 2013. doi:10.5902/21754977

D'Almeida, N.; Lins Peliz, A. C. Le changement climatique en image et en texte. Recherches en Communication, 35,
17-36, 2011. Disponível em: <http://sites.uclouvain.be/rec/ index.php/rec/article/view/7334/6463>.

Dupuy, J.-P. Pour un catastrophisme éclairé. Paris: Éditions du Seuil, 2002.

Dupuy, J.-P. Petite métaphysique des tsunamis. Paris: Seuil, 2005. Version électronique Kindle.

Guillemot, H. Les modèles numériques de climat, In: Les modèles du futur. Paris: La Découverte, 2007. p. 91-112.

Hamilton, C. Les apprentis sorciers du climat. Raisons et déraisons de la géo-ingénierie. Paris: Seuil, 2013. Version électronique pour Kindle.

Jonas, H. Le principe responsabilité. Une éthique pour la civilisation technologique. Paris: Flamarion, 1998.

Jonas, H. Une éthique pour la nature. Paris: Desclée de Brouwer, 2000.

Mauger-Parat, M.; Lins Peliz, A. C. Expertise, controverse, polémique: trois formes de confrontation pour aborder le débat sur le changement climatique dans la presse française. Vertigo - La Revue Electronique en Sciences de l'Environnement, 13(2), 2013. Disponível em: < http://vertigo.revues. org/14297>.

Micheau, B. Le changement climatique dans la presse magazine: expliquer la menace, impliquer les individus, prédire la catastrophe. Communication et Langages, 172, 27-51, 2012.

Miguel, K. Os paradigmas da imprensa na cobertura das políticas ambientais. Intercom - RBCC, 35(1), 111-131, 2012.

Miranda Costa, L. O esverdeamento da imprensa. Estudos em Jornalismo e Mídia, 3(2), 41-54, 2006. Disponível em: $<$ https://periodicos.ufsc.br/index.php/jornalismo/article/ download/2289/2017>.

Moirand, S. Les discours de la presse quotidienne. Observer, analyser, comprendre. Paris: PUF, 2011.

More, T. L'Utopie. Domaine Public, Bibebook, 1517.

Mormont, M.; Dasnoy, C. Expertise scientifique et action publique: le cas du changement climatique dans trois pays européens. Nature, Sciences et Société, 1(3), 16-25, 1995. Disponível em: <http://benhur.teluq.uquebec.ca/SPIP/ 
env6003/IMG/pdf/module5/Texte_Expertise_Scientifique. pdf $>$.

Painter, J.; Ashe, T. Cross-national comparison of the presence of climate scepticism in the print media in six countries, 2007-10. Environmental Research Letters Journal, 7(4), 2012.

Péquignot, B. Karl Marx: l'utopie, la raison et la sience, Quaderni, 40, 97-111, 1999. Disponível em: $<$ http://www. persee.fr/doc/quad_0987-1381_1999_num_40_1_1430>.

Rabeharisoa, V. Science, politique et grand public. La médiatisation du risque climatique. Sciences de la Société, 41, 19-39, 1997.

Stableford, B. Ecology and dystopia. In: Claeys, G. (Ed.). The Cambridge companion to utopian literature. Cambridge: Cambridge University Press, 2010. p. 259-266.

Todorov, T. Mikhaïl Bakhtine. Le principe dialogique, suivi des Écrits du Cercle de Bakhtine. Paris: Minuit, 1981. Version électronique pour Kindle.
Tourinho Girardi, I. M. Discursos e vozes por detrás da Cop-15 e Cop-16. In: Anais do $9^{\circ}$ Encontro Nacional de Pesquisadores em Jornalismo. Rio de Janeiro, Eco-Universidade Federal do Rio de Janeiro, 2011. Disponível em: <http://sbpjor.kamotini.kinghost.net/sbpjor/admjor/ arquivos/9encontro/CC_19.pdf>.

Tourinho Girardi, I. M.; Herte de Moraes, C.; Beling Loose, E. Bases do jornalismo ambiental e os desafios para a cobertura da Rio+20. Razón y Palabra, 79, 2012. Disponível em: $<$ http://www.razonypalabra.org.mx/N/N79/M79/01_TourinhoHerteBeling_M79.pdf>.

Van Dijk, T. La noticia como discurso. Comprensión, estructura y producción de la información. Barcelona: Paidós, 1990.

Vieira, F. The concept of utopia. In: Clayes, G. (Éd.). The Cambridge companion to utopian literature. Cambridge: Cambridge University Press, 2010. p. 3-27. 